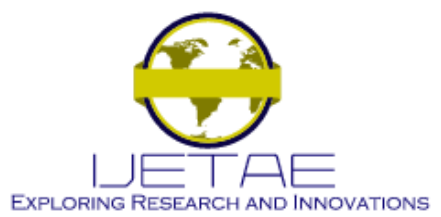

International Journal of Emerging Technology and Advanced Engineering

Website: www.ijetae.com (ISSN 2250-2459, ISO 9001:2008 Certified Journal, Volume 10, Issue 07, July 2020)

\title{
Vibration Analysis of the Tool Bearing Spindle in Case of Superfinishing Technological Processes. Insertion of External Strains and Determining the Balance Conditions
}

\author{
Daniel Popescu \\ Faculty of Mechanics, University of Craiova, Calea Bucuresti, No. 165, Craiova, Romania;
}

\begin{abstract}
The paper presents the main spindle model at superfinishing operations taking into account the external strains determined by the resulting forces and moments in the grinding process. An important role is played by the geometric configuration of the main spindle. The initial conditions of the movement are presented for each geometric section. There are also emphasized the inertial forces and moments determined by the tool position. There are also established the balance conditions, situation in which the spindle is deformed but without vibrating. There are computed the expressions for the cutting forces, bending moments as well as the continuity equations regarding spindle deformation in the considered sections.
\end{abstract}

Keywords - Balance conditions, eccentric, external strains, main spindle, superfinishing.

\section{INTRODUCTION}

Establishing the general movement equations that describe the evolution of the vibration movement of the tool bearing spindle in case of superfinishing operations emphasizes the propagation of the oscillating movement along the coordinate axes. Their establishment was done with respect to a proper reference system attached to the main spindle.

For determining the external strains it is necessary to know the specific work conditions of the spindle in case of superfinishing.

In this context, the geometric configuration of the spindle plays a major role [1]. It is required to transpose the movement equations with respect to a fixed reference system. Thus can be evaluated the optimal movement conditions $[2,3]$.

\section{INSERTION OF THE EXTERNAL STRAINS}

The analyzed tool bearing spindle model is given in fig. 1:

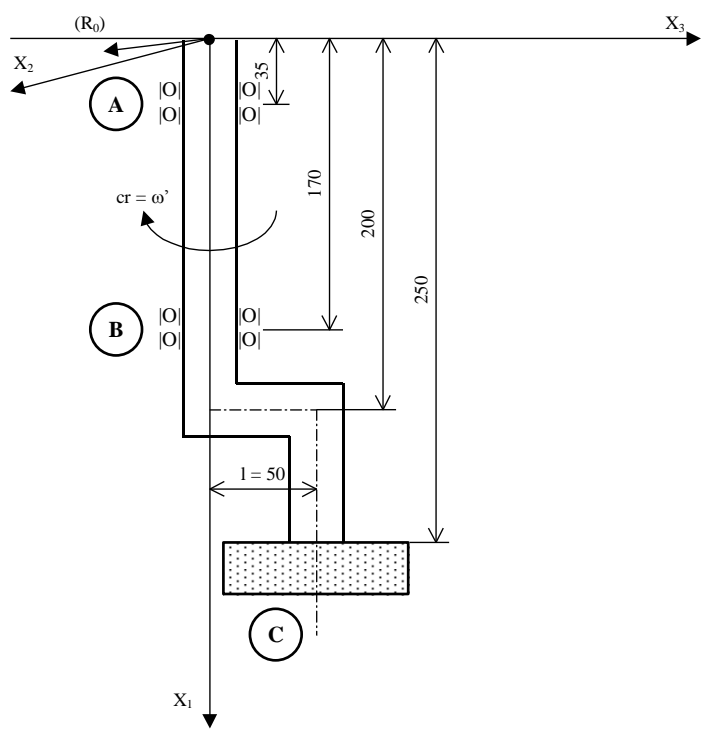

Figure 1. Ideal model of the spindle

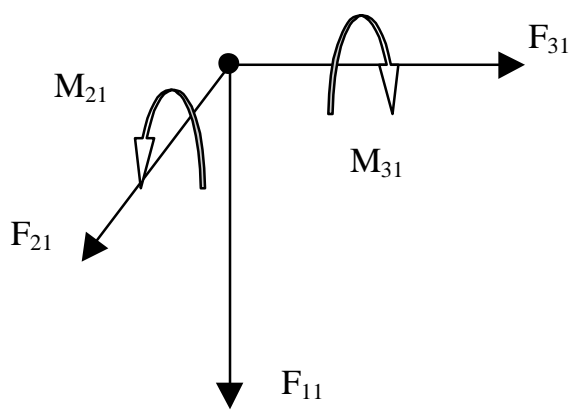

Figure 2. Action of bearing upon spindle

Corresponding to the upper bearing $\mathrm{A}$, the reactions of the bearing upon the eccentric spindle are given in fig. 2 . 


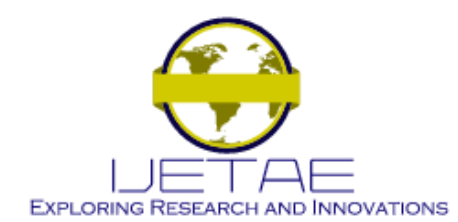

International Journal of Emerging Technology and Advanced Engineering

Website: www.ijetae.com (ISSN 2250-2459, ISO 9001:2008 Certified Journal, Volume 10, Issue 07, July 2020)

The forces, moments and impulses for the two bearings are given by:

$$
\begin{aligned}
& \{\mathrm{p}\}^{(1)}=\left\{\begin{array}{l}
\mathrm{F}_{11} \\
\mathrm{~F}_{21} \\
\mathrm{~F}_{31}
\end{array}\right\} \delta\left(\mathrm{x}_{1}-30\right) \\
& \{\mathrm{m}\}^{(1)}=\left\{\begin{array}{c}
0 \\
\mathrm{M}_{21} \\
\mathrm{M}_{31}
\end{array}\right\} \delta\left(\mathrm{x}_{1}-30\right) \\
& \{\mathrm{p}\}^{(2)}=\left\{\begin{array}{l}
\mathrm{F}_{12} \\
\mathrm{~F}_{22} \\
\mathrm{~F}_{32}
\end{array}\right\} \delta\left(\mathrm{x}_{1}-170\right) \\
& \{\mathrm{m}\}^{(2)}=\left\{\begin{array}{c}
0 \\
\mathrm{M}_{22} \\
\mathrm{M}_{32}
\end{array}\right\} \delta\left(\mathrm{x}_{1}-170\right)
\end{aligned}
$$$$
\text { where [4]: } \quad \delta\left(\mathrm{x}_{1}-\mathrm{l}_{\mathrm{i}}\right)=\left\{\begin{array}{l}
1, \mathrm{x}_{1}=\mathrm{1}_{\mathrm{i}} \\
0, \mathrm{x}_{1} \neq \mathrm{1}_{\mathrm{i}}
\end{array}\right.
$$

The bearings produce a damping whose expression is:

$$
\{p\}^{(a)}=-\left\{\begin{array}{l}
\mathrm{C}_{1} \dot{\mathrm{u}}_{1} \\
\mathrm{C}_{2} \dot{\mathrm{u}}_{2} \\
\mathrm{C}_{3} \dot{\mathrm{u}}_{3}
\end{array}\right\}\left[\delta\left(\mathrm{x}_{1}-30\right)+\delta\left(\mathrm{x}_{1}-170\right)\right]
$$

In stabilized regime, the movement equations become:

$$
\begin{aligned}
& \rho A \ddot{u}_{1}-\mathrm{EAu}_{1,11}=\mathrm{F}_{11} \delta\left(\mathrm{x}_{1}-30\right)+\mathrm{F}_{12} \delta\left(\mathrm{x}_{1}-170\right)- \\
& -\mathrm{c}_{1} \dot{\mathrm{u}}_{1}\left[\delta\left(\mathrm{x}_{1}-30\right)+\delta\left(\mathrm{x}_{1}-170\right)\right] \\
& \rho A \ddot{\mathrm{u}}_{3}-\rho \ddot{\mathrm{u}}_{3,11}+2 \rho \mathrm{A}\left(\Omega+\omega^{\prime}\right) \dot{\mathrm{u}}_{2}-\rho \mathrm{A}\left(\Omega+\omega^{\prime}\right)^{2} \mathrm{u}_{3}+ \\
+ & \mathrm{EIu}_{3,111}=\mathrm{M}_{21} \delta\left(\mathrm{x}_{1}-30\right)+\mathrm{M}_{22} \delta\left(\mathrm{x}_{1}-170\right)+ \\
+ & \mathrm{F}_{31} \delta\left(\mathrm{x}_{1}-30\right)+\mathrm{F}_{32} \delta\left(\mathrm{x}_{1}-170\right)-\mathrm{c}_{3} \dot{\mathrm{u}}_{3}\left[\delta\left(\mathrm{x}_{1}-30\right)+\right. \\
& \left.+\delta\left(\mathrm{x}_{1}-30\right)\right] \\
- & \rho \mathrm{A} \ddot{\mathrm{u}}_{2}+\rho \mathrm{I} \ddot{\mathrm{u}}_{2,11}+2 \rho \mathrm{A}\left(\Omega+\omega^{\prime}\right) \dot{\mathrm{u}}_{3}+\rho \mathrm{A}\left(\Omega+\omega^{\prime}\right)^{2} \mathrm{u}_{2}- \\
- & \mathrm{EIu_{2,1111 }}=\mathrm{M}_{31} \delta\left(\mathrm{x}_{1}-30\right)+\mathrm{M}_{32} \delta\left(\mathrm{x}_{1}-170\right)- \\
- & \mathrm{F}_{21} \delta\left(\mathrm{x}_{1}-30\right)-\mathrm{F}_{22} \delta\left(\mathrm{x}_{1}-170\right)-\mathrm{c}_{2} \dot{\mathrm{u}}_{2}\left[\delta\left(\mathrm{x}_{1}-30\right)+\right. \\
+ & \left.\delta\left(\mathrm{x}_{1}-170\right)\right]
\end{aligned}
$$

Also, the movement of the abrasive tools will produce:

$$
\begin{aligned}
& \overrightarrow{\mathrm{F}}_{\mathrm{in}}=-\mathrm{m}_{\mathrm{T}} \cdot \overrightarrow{\mathrm{a}}_{\mathrm{c}}=\mathrm{m}_{\mathrm{T}}\left(\ddot{\mathrm{u}}_{1} \overrightarrow{\mathrm{i}}_{1}+\ddot{\mathrm{u}}_{2} \overrightarrow{\mathrm{i}}_{2}+\ddot{\mathrm{u}}_{3} \overrightarrow{\mathrm{i}}_{3}\right) \\
& \overrightarrow{\mathrm{M}}_{\mathrm{in}}=\left[\mathrm{J}_{\mathrm{d}} \ddot{\mathrm{u}}_{3,1}-\left(\mathrm{J}_{0}-\mathrm{J}_{\mathrm{d}}\right)\left(\Omega+\omega^{\prime}\right) \dot{\mathrm{u}}_{2,1}\right] \overrightarrow{\mathrm{i}}_{2}- \\
& -\left[\mathrm{J}_{\mathrm{d}} \ddot{\mathrm{u}}_{2,1}-\left(\mathrm{J}_{0}-\mathrm{J}_{\mathrm{d}}\right)\left(\Omega+\omega^{\prime}\right) \dot{\mathrm{u}}_{3,1}\right] \overrightarrow{\mathrm{i}}_{3}
\end{aligned}
$$

The use of abrasive tool introduces specific elements. In this case, the limit conditions will be:

$$
\begin{gathered}
\mathrm{EAu}_{1,1}=0 \\
\mathrm{EIu}_{2,111}-\rho \ddot{I u}_{2,1}-\mathrm{EAu}_{1,1} \mathrm{u}_{2,1}=0
\end{gathered}
$$

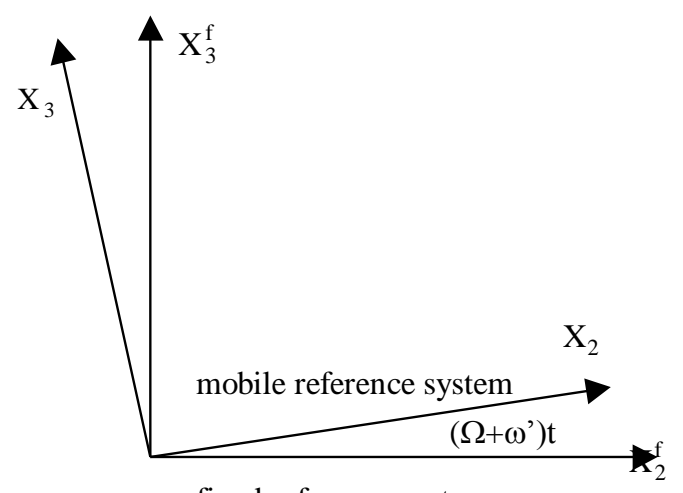

fixed reference system

Figure 3. The position of the fixed reference system with respect to the mobile reference system

$$
\mathrm{EIu}_{3,111}-\rho \ddot{I u}_{3,1}-\mathrm{EAu}_{1,1} \mathrm{u}_{3,1}=0
$$

$$
\mathrm{EIu}_{2,11}=0
$$

$$
\mathrm{EIu}_{3,11}=0
$$

$\mathrm{EAu}_{1,1}+\mathrm{m}_{\mathrm{p}} \ddot{\mathrm{u}}_{1}=\mathrm{R}_{1}$

$$
\begin{gathered}
\mathrm{EIu}_{2,111}-\rho \ddot{\mathrm{u}}_{2,1}-\mathrm{EAu}_{1,1} \mathrm{u}_{2,1}-\mathrm{m}_{\mathrm{p}} \ddot{\mathrm{u}}_{2}=-\mathrm{R}_{2} \\
\mathrm{EIu}_{3,111}-\rho \ddot{\mathrm{u}}_{3,1}-\mathrm{EAu}_{1,1} \mathrm{u}_{3,1}-\mathrm{m}_{\mathrm{p}} \ddot{\mathrm{u}}_{3}=-\mathrm{R}_{3} \\
\mathrm{EIu}_{3,11}+\mathrm{J}_{\mathrm{d}} \ddot{\mathrm{u}}_{3,1}-\left(\mathrm{J}_{0}-\mathrm{J}_{\mathrm{d}}\right)\left(\Omega+\omega^{\prime}\right) \dot{\mathrm{u}}_{2,1}=-\mathrm{M}_{\mathrm{R} 2} \\
\mathrm{EIu}_{2,11}+\mathrm{J}_{\mathrm{d}} \ddot{\mathrm{u}}_{2,1}+\left(\mathrm{J}_{0}-\mathrm{J}_{\mathrm{d}}\right)\left(\Omega+\omega^{\prime}\right) \dot{\mathrm{u}}_{3,1}=\mathrm{M}_{\mathrm{R} 3}
\end{gathered}
$$

in which:

$$
\mathrm{m}_{\mathrm{T}}-\text { mass of abrasive tool }
$$




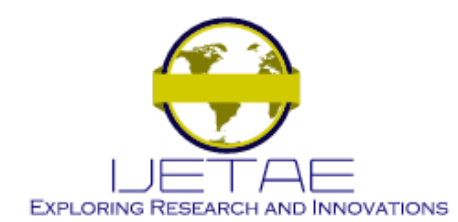

\section{International Journal of Emerging Technology and Advanced Engineering \\ Website: www.ijetae.com (ISSN 2250-2459, ISO 9001:2008 Certified Journal, Volume 10, Issue 07, July 2020)}

$\mathrm{J}_{0}$ - inertial moment of the grinding wheel with respect to the symmetry axis;

$\mathrm{J}_{\mathrm{d}}$ - inertial moment of the grinding wheel with respect to a diametral axis;

$R_{2}, R_{3}, M_{R 2}, M_{R 3}$ - components of the external force and moment produced by the abrasive process.

Observation: Since the deformations of the main spindle with respect to the proper reference system of the spindle cannot be measured due to the rotation movement, they are projected on the axes of a fixed reference system.

The relations for transforming the fixed system coordinates are:

$$
[Q]=\left|\begin{array}{ccc}
1 & 0 & 0 \\
0 & \cos \left(\Omega+\omega^{\prime}\right) & -\sin \left(\Omega+\omega^{\prime}\right) \\
0 & \sin \left(\Omega+\omega^{\prime}\right) & \cos \left(\Omega+\omega^{\prime}\right)
\end{array}\right|
$$

This represents the reference system change matrix.

If we denote by $\vec{v}=v_{1} \vec{i}_{1 f}+v_{2} \vec{i}_{2 f}+v_{3} \vec{i}_{3 f}$, the point displacement on the spindle axis can be written as:

$$
\left\{\begin{array}{l}
\mathrm{v}_{1} \\
\mathrm{v}_{2} \\
\mathrm{v}_{3}
\end{array}\right\}=\left|\begin{array}{ccc}
1 & 0 & 0 \\
0 & \cos \left(\Omega+\omega^{\prime}\right) & -\sin \left(\Omega+\omega^{\prime}\right) \\
0 & \sin \left(\Omega+\omega^{\prime}\right) & \cos \left(\Omega+\omega^{\prime}\right)
\end{array}\right|\left\{\begin{array}{l}
\mathrm{u}_{1} \\
\mathrm{u}_{2} \\
\mathrm{u}_{3}
\end{array}\right\}
$$

and, conversely,

$$
\left\{\begin{array}{l}
\mathrm{u}_{1} \\
\mathrm{u}_{2} \\
\mathrm{u}_{3}
\end{array}\right\}=\left|\begin{array}{ccc}
1 & 0 & 0 \\
0 & \cos \left(\Omega+\omega^{\prime}\right) & \sin \left(\Omega+\omega^{\prime}\right) \\
0 & -\sin \left(\Omega+\omega^{\prime}\right) & \cos \left(\Omega+\omega^{\prime}\right)
\end{array}\right|\left\{\begin{array}{c}
\mathrm{v}_{1} \\
\mathrm{v}_{2} \\
\mathrm{v}_{3}
\end{array}\right\}
$$

In more detail:

$$
\begin{gathered}
\mathrm{u}_{1}=\mathrm{v}_{1} \quad \mathrm{u}_{1,1}=\mathrm{v}_{1,1} \\
\dot{\mathrm{u}}_{1}=\dot{\mathrm{v}}_{1} \quad \mathrm{u}_{1,11}=\mathrm{v}_{1,11} \\
\ddot{\mathrm{u}}_{1}=\ddot{\mathrm{v}}_{1} \\
\mathrm{u}_{2}=\cos \left(\Omega+\omega^{\prime}\right) \mathrm{tv}_{2}+\sin \left(\Omega+\omega^{\prime}\right) \mathrm{tv}_{3} \\
\dot{\mathrm{u}}_{2}=\cos \left(\Omega+\omega^{\prime}\right) \mathrm{t}\left(\dot{\mathrm{v}}_{2}+\left(\Omega+\omega^{\prime}\right) \mathrm{v}_{3}\right)+ \\
+\sin \left(\Omega+\omega^{\prime}\right) \mathrm{t}\left(\dot{\mathrm{v}}_{3}-\left(\Omega+\omega^{\prime}\right) \mathrm{v}_{2}\right) \\
\ddot{\mathrm{u}}_{2}=\cos \left(\Omega+\omega^{\prime}\right) \mathrm{t}\left(\ddot{\mathrm{v}}_{2}+2\left(\Omega+\omega^{\prime}\right) \dot{\mathrm{v}}_{3}-\left(\Omega+\omega^{\prime}\right)^{2} \mathrm{v}_{2}\right)+ \\
+\sin \left(\Omega+\omega^{\prime}\right) \mathrm{t}\left(\ddot{\mathrm{v}}_{3}+2\left(\Omega+\omega^{\prime}\right) \dot{\mathrm{v}}_{2}-\left(\Omega+\omega^{\prime}\right)^{2} \mathrm{v}_{3}\right) \\
\mathrm{u}_{2,1}=\cos \left(\Omega+\omega^{\prime}\right) \mathrm{tv}_{2,1}+\sin \left(\Omega+\omega^{\prime}\right) \mathrm{tv}_{3,1}
\end{gathered}
$$

$$
\begin{gathered}
\mathrm{u}_{2,11}=\cos \left(\Omega+\omega^{\prime}\right) \mathrm{tv}_{2,11}+\sin \left(\Omega+\omega^{\prime}\right) \mathrm{tv}_{3,11} \\
\mathrm{u}_{2,1111}=\cos \left(\Omega+\omega^{\prime}\right) \mathrm{tv}_{2,1111}+\sin \left(\Omega+\omega^{\prime}\right) \mathrm{tv}_{3,1111} \\
\ddot{\mathrm{u}}_{2,11}=\cos \left(\Omega+\omega^{\prime}\right) \mathrm{t}\left(\ddot{\mathrm{v}}_{2,11}+2\left(\Omega+\omega^{\prime}\right) \dot{\mathrm{v}}_{3,11}-\left(\Omega+\omega^{\prime}\right)^{2} \mathrm{v}_{2,11}\right)+ \\
+\sin \left(\Omega+\omega^{\prime}\right) \mathrm{t}\left(\ddot{\mathrm{v}}_{3,11}-2\left(\Omega+\omega^{\prime}\right) \dot{\mathrm{v}}_{2,11}-\left(\Omega+\omega^{\prime}\right)^{2} \mathrm{v}_{3,11}\right) \\
\mathrm{u}_{3}=-\sin \left(\Omega+\omega^{\prime}\right) \mathrm{t} \mathrm{v}_{2}+\cos \left(\Omega+\omega^{\prime}\right) \mathrm{tv}_{3} \\
\mathrm{u}_{3,1111}=-\sin \left(\Omega+\omega^{\prime}\right) \mathrm{t} \mathrm{v}_{2,1111}+\cos \left(\Omega+\omega^{\prime}\right) \mathrm{t} \mathrm{v}_{3,1111} \\
\dot{\mathrm{u}}_{3}=\cos \left(\Omega+\omega^{\prime}\right) \mathrm{t}\left(\dot{\mathrm{v}}_{3}-\left(\Omega+\omega^{\prime}\right) \mathrm{v}_{2}\right)- \\
-\sin \left(\Omega+\omega^{\prime}\right) \mathrm{t}\left(\dot{\mathrm{v}}_{2}-\left(\Omega+\omega^{\prime}\right) \mathrm{v}_{3}\right) \\
\ddot{\mathrm{u}}_{3}=\cos \left(\Omega+\omega^{\prime}\right) \mathrm{t}\left(\ddot{\mathrm{v}}_{3}-2\left(\Omega+\omega^{\prime}\right) \dot{\mathrm{v}}_{2}-\left(\Omega+\omega^{\prime}\right)^{2} \mathrm{v}_{3}\right)- \\
-\sin \left(\Omega+\omega^{\prime}\right) \mathrm{t}\left(\ddot{\mathrm{v}}_{2}+2\left(\Omega+\omega^{\prime}\right) \dot{\mathrm{v}}_{3}-\left(\Omega+\omega^{\prime}\right)^{2} \mathrm{v}_{2}\right) \\
\ddot{\mathrm{u}}_{3,11}=\cos \left(\Omega+\omega^{\prime}\right) \mathrm{t}\left(\ddot{\mathrm{v}}_{3,11}-2\left(\Omega+\omega^{\prime}\right) \dot{\mathrm{v}}_{2,11}-\left(\Omega+\omega^{\prime}\right)^{2} \mathrm{v}_{3,11}\right)- \\
-\sin \left(\Omega+\omega^{\prime}\right) \mathrm{t}\left(\ddot{\mathrm{v}}_{2,11}+2\left(\Omega+\omega^{\prime}\right) \dot{\mathrm{v}}_{3,11}-\left(\Omega+\omega^{\prime}\right)^{2} \mathrm{v}_{2,11}\right)
\end{gathered}
$$

The forces and torques in the reference system are given by:

$$
\begin{gathered}
\mathrm{F}_{11}=\mathrm{F}_{11}^{\mathrm{f}} \\
\mathrm{F}_{21}=\cos \left(\Omega+\omega^{\prime}\right) \mathrm{tF}_{21}^{\mathrm{f}}+\sin \left(\Omega+\omega^{\prime}\right) \mathrm{tF}_{31}^{\mathrm{f}} \\
\mathrm{F}_{31}=-\sin \left(\Omega+\omega^{\prime}\right) \mathrm{tF}_{21}^{\mathrm{f}}+\cos \left(\Omega+\omega^{\prime}\right) \mathrm{tF}_{31}^{\mathrm{f}} \\
\mathrm{M}_{21}=\cos \left(\Omega+\omega^{\prime}\right) \mathrm{tM}_{21}^{\mathrm{f}}+\sin \left(\Omega+\omega^{\prime}\right) \mathrm{tM}_{31}^{\mathrm{f}} \\
\mathrm{M}_{31}=-\sin \left(\Omega+\omega^{\prime}\right) \mathrm{tM}_{21}^{\mathrm{f}}+\cos \left(\Omega+\omega^{\prime}\right) \mathrm{tM}_{31}^{\mathrm{f}}
\end{gathered}
$$

The movement equations become:

$$
\begin{aligned}
& \rho A \ddot{v}_{1}-\operatorname{EAv}_{1,11}=\mathrm{F}^{\mathrm{f}}{ }_{11} \delta\left(\mathrm{x}_{1}-30\right)+\mathrm{F}^{\mathrm{f}}{ }_{12} \delta\left(\mathrm{x}_{1}-170\right)- \\
& -\mathrm{c}^{\mathrm{f}}{ }_{1} \dot{\mathrm{v}}_{1}\left[\delta\left(\mathrm{x}_{1}-30\right)+\delta\left(\mathrm{x}_{1}-170\right)\right] \\
& \rho A \ddot{v}_{3}-\rho \mathrm{I} \dot{\mathrm{v}}_{3,11}+2 \rho \mathrm{I}\left(\Omega+\omega^{\prime}\right) \dot{\mathrm{v}}_{2,11}+\rho \mathrm{I}\left(\Omega+\omega^{\prime}\right)^{2} \mathrm{v}_{3,11}+ \\
& +\operatorname{EIv}_{3,1111}=\mathrm{M}^{\mathrm{f}}{ }_{21} \delta\left(\mathrm{x}_{1}-30\right)+\mathrm{M}^{\mathrm{f}}{ }_{22} \delta\left(\mathrm{x}_{1}-170\right)+ \\
& +\mathrm{F}^{\mathrm{f}}{ }_{31} \delta\left(\mathrm{x}_{1}-30\right)+\mathrm{F}^{\mathrm{f}}{ }_{32} \delta\left(\mathrm{x}_{1}-170\right)-\mathrm{c}^{\mathrm{f}}{ }_{3} \dot{\mathrm{v}}_{3}\left[\delta\left(\mathrm{x}_{1}-30\right)+\right. \\
& \left.+\delta\left(\mathrm{x}_{1}-170\right)\right] \\
& \rho \mathrm{A} \ddot{\mathrm{v}}_{2}+\rho \mathrm{I} \ddot{\mathrm{v}}_{2,11}+2 \rho \mathrm{I}\left(\Omega+\omega^{\prime}\right) \dot{\mathrm{v}}_{3,11}-\rho \mathrm{I}\left(\Omega+\omega^{\prime}\right)^{2} \mathrm{v}_{2,11}- \\
& -\operatorname{EIv}_{2,1111}=\mathrm{M}^{\mathrm{f}}{ }_{31} \delta\left(\mathrm{x}_{1}-30\right)+\mathrm{M}^{\mathrm{f}}{ }_{32} \delta\left(\mathrm{x}_{1}-170\right)- \\
& -\mathrm{F}^{\mathrm{f}}{ }_{21} \delta\left(\mathrm{x}_{1}-30\right)-\mathrm{F}^{\mathrm{f}}{ }_{22} \delta\left(\mathrm{x}_{1}-170\right)+\mathrm{c}^{\mathrm{f}}{ }_{2} \dot{\mathrm{v}}_{2}\left[\delta\left(\mathrm{x}_{1}-30\right)+\right. \\
& \left.+\delta\left(\mathrm{x}_{1}-170\right)\right]
\end{aligned}
$$

The limit conditions will be: 


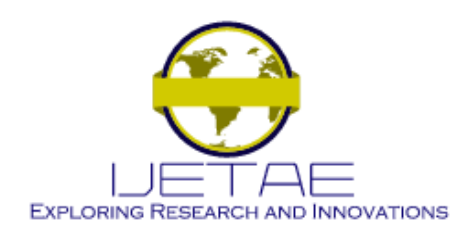

International Journal of Emerging Technology and Advanced Engineering

Website: www.ijetae.com (ISSN 2250-2459, ISO 9001:2008 Certified Journal, Volume 10, Issue 07, July 2020)

$$
\mathrm{EAv}_{1,1}=0
$$

$\operatorname{EIv}_{2,111}-\rho \mathrm{I} \ddot{\mathrm{v}}_{2,1}-2 \rho \mathrm{I}\left(\Omega+\omega^{\prime}\right) \dot{\mathrm{v}}_{3,1}+\rho \mathrm{I}\left(\Omega+\omega^{\prime}\right)^{2} \mathrm{v}_{2,1}-$

$-\mathrm{EAv}_{1,1} \mathrm{v}_{2,1}=0$

$\mathrm{EIv}_{3,111}-\rho \mathrm{I} \ddot{\mathrm{v}}_{3,1}+2 \rho \mathrm{I}\left(\Omega+\omega^{\prime}\right) \dot{\mathrm{v}}_{2,1}+\rho \mathrm{I}\left(\Omega+\omega^{\prime}\right)^{2} \mathrm{v}_{3,1}-$

$-\mathrm{EAv}_{1,1} \mathrm{v}_{3,1}=0$

$$
\begin{gathered}
\operatorname{EIv}_{2,11}=0 \\
\operatorname{EIv}_{3,11}=0 \\
\operatorname{EAv}_{1,1}+\mathrm{m}_{\mathrm{p}} \ddot{\mathrm{v}}_{1}=\mathrm{R}_{1}^{\mathrm{f}} \\
\operatorname{EIv}_{2,111}-\rho \ddot{\mathrm{v}}_{2,1}+2 \rho \mathrm{I}\left(\Omega+\omega^{\prime}\right) \dot{\mathrm{v}}_{3,1}+\rho \mathrm{I}\left(\Omega+\omega^{\prime}\right)^{2} \mathrm{v}_{2,1}- \\
\mathrm{EAv}_{1,1} \mathrm{v}_{2,1}-\mathrm{m}_{\mathrm{p}}\left(\ddot{\mathrm{v}}_{2}+2\left(\Omega+\omega^{\prime}\right) \dot{\mathrm{v}}_{3}-\left(\Omega+\omega^{\prime}\right)^{2} \mathrm{v}_{2}\right)= \\
=-\mathrm{R}_{2}^{\mathrm{f}} \\
\operatorname{EIv}_{3,111}-\rho \ddot{\mathrm{v}}_{3,1}+2 \rho \mathrm{I}\left(\Omega+\omega^{\prime}\right) \dot{\mathrm{v}}_{2,1}+\rho \mathrm{I}\left(\Omega+\omega^{\prime}\right)^{2} \mathrm{v}_{3,1}- \\
\operatorname{EAv}_{1,1} \mathrm{v}_{3,1}-\mathrm{m}_{\mathrm{p}}\left(\ddot{\mathrm{v}}_{3}+2\left(\Omega+\omega^{\prime}\right) \dot{\mathrm{v}}_{2}-\left(\Omega+\omega^{\prime}\right)^{2} \mathrm{v}_{3}\right)= \\
=-\mathrm{R}_{3}^{\mathrm{f}} \\
\operatorname{EIv}_{3,11}+\mathrm{J}_{\mathrm{d}}\left(\ddot{\mathrm{v}}_{3,1}+2\left(\Omega+\omega^{\prime}\right) \dot{\mathrm{v}}_{2,1}-\left(\Omega+\omega^{\prime}\right)^{2} \mathrm{v}_{3,1}\right)+ \\
+\left(\mathrm{J}_{0}-\mathrm{J}_{\mathrm{d}}\right)\left(\Omega+\omega^{\prime}\right) \cdot\left(\dot{\mathrm{v}}_{2,1}-\mathrm{v}_{3,1}\right)=-\mathrm{M}_{\mathrm{R} 2}^{\mathrm{f}} \\
\operatorname{EIv}_{2,11}+\mathrm{J}_{\mathrm{d}}\left(\ddot{\mathrm{v}}_{2,1}+2\left(\Omega+\omega^{\prime}\right) \dot{\mathrm{v}}_{3,1}-\left(\Omega+\omega^{\prime}\right)^{2} \mathrm{v}_{3,1}\right)+ \\
+\left(\mathrm{J}_{0}-\mathrm{J}_{\mathrm{d}}\right)\left(\Omega+\omega^{\prime}\right)\left(\dot{\mathrm{v}}_{3,1}-\left(\Omega+\omega^{\prime}\right) \mathrm{v}_{2,1}\right)=\mathrm{M}_{\mathrm{R} 3}^{\mathrm{f}}
\end{gathered}
$$

In order to establish the balance configuration, we consider that on the ensemble spindle-bearings-tool actions a system of external actions that produces a deformation of the spindle, without causing it to vibrate [5].

At balance, the initial conditions become:

$$
\begin{aligned}
& \operatorname{EAv}_{1,11}=0 \\
& \rho \mathrm{I}\left(\Omega+\omega^{\prime}\right)^{2} \mathrm{v}_{2,11}+\operatorname{EIv}_{2,1111}=0 \\
& \rho \mathrm{I}\left(\Omega+\omega^{\prime}\right)^{2} v_{3,11}+\operatorname{EIv}_{3,1111}=0
\end{aligned}
$$

and:

$$
\begin{gathered}
x_{1}=0 \\
\operatorname{EAv}_{1,1}^{(0)}(0)=0 \\
\operatorname{EIv}_{2,111}^{(0)}(0)-\rho I\left(\Omega+\omega^{\prime}\right)^{2} v_{2,1}^{(0)}(0)-\operatorname{EAv}_{1,1}^{(0)}(0) v_{2,1}^{(0)}(0)=0 \\
\operatorname{EIv}_{3,111}^{(0)}(0)-\rho I\left(\Omega+\omega^{\prime}\right)^{2} v_{3,1}^{(0)}(0)-\operatorname{EAv}_{1,1}^{(0)}(0) v_{3,1}^{(0)}(0)=0
\end{gathered}
$$

$$
\begin{aligned}
& \operatorname{EIv}_{2,11}^{(0)}(0)=0 \\
& \operatorname{EIv}_{3,11}^{(0)}(0)=0 \\
& \mathrm{x}_{1}=30: \mathrm{x}_{1}=170 \\
& {\left[\operatorname{EAv}_{1,1}\right]_{\mathrm{x}_{1}=30^{+} ; 170^{+}}=\mathrm{F}_{\mathrm{ij}}^{\mathrm{f}_{1}^{*}=30^{-} ; 170^{*}}} \\
& \left|\operatorname{EIv}_{2,111}+\rho \mathrm{I}\left(\Omega+\omega^{\prime}\right)^{2} \mathrm{v}_{2,1}-\operatorname{EAv}_{1,1} \mathrm{v}_{2,1}\right|_{\mathrm{x}_{1}=30^{+} ; 170^{+}}= \\
& =-\mathrm{F}_{\mathrm{i}+1, \mathrm{j}}^{\mathrm{f} *} \\
& \left|\operatorname{EIv}_{3,111}+\rho \mathrm{I}\left(\Omega+\omega^{\prime}\right)^{2} \mathrm{v}_{3,1}-\operatorname{EAv}_{1,1} \mathrm{v}_{3,1}\right|_{\mathrm{x}_{1}=30^{+} ; 170^{+}}= \\
& =-\mathrm{F}_{\mathrm{i}+2, \mathrm{j}}^{\mathrm{f} *} \\
& {\left[\operatorname{EAv}_{3,11}\right]_{\mathrm{x}_{1}=30^{+} ; 170^{+}}=-\mathbf{M}_{\mathrm{i}+1, \mathrm{j}}^{\mathrm{f*}}} \\
& {\left[\operatorname{EAv}_{2,11}\right]_{\mathrm{x}_{1}=30^{+} ; 170^{+}}=\mathrm{M}_{\mathrm{i}+2, \mathrm{j}}^{\mathrm{f*}}} \\
& \mathrm{v}_{1}^{(0,1)}\left(\mathrm{x}_{1}\right)=\mathrm{v}_{1}^{(1,2)}\left(\mathrm{x}_{1}\right) \\
& \mathrm{v}_{2}^{(0,1)}\left(\mathrm{x}_{1}\right)=\mathrm{v}_{2}^{(1,2)}\left(\mathrm{x}_{1}\right) \\
& \mathrm{v}_{3}^{(0,1)}\left(\mathrm{x}_{1}\right)=\mathrm{v}_{3}^{(1,2)}\left(\mathrm{x}_{1}\right) \\
& \mathrm{v}_{2,1}^{(0,1)}\left(\mathrm{x}_{1}\right)=\mathrm{v}_{2,1}^{(1,2)}\left(\mathrm{x}_{1}\right) \\
& \mathrm{v}_{3,1}^{(0,1)}\left(\mathrm{x}_{1}\right)=\mathrm{v}_{3,1}^{(1,2)}\left(\mathrm{x}_{1}\right) \\
& \mathrm{x}_{1}=250 \\
& \operatorname{EAv}_{1,1}^{(2)}\left(\mathrm{x}_{1}\right)=\mathrm{R}_{1}^{\mathrm{f} *} \\
& \operatorname{EIv}_{2,111}^{(2)}\left(\mathrm{x}_{1}\right)+\rho \mathrm{I}\left(\Omega+\omega^{\prime}\right)^{2} \mathrm{v}_{2,1}^{(2)}\left(\mathrm{x}_{1}\right)- \\
& -\operatorname{EAv}_{1,1}^{(2)}\left(\mathrm{x}_{1}\right) \mathrm{v}_{2,1}^{(2)}\left(\mathrm{x}_{1}\right)+\mathrm{m}_{\mathrm{p}} \Omega^{2} \mathrm{v}_{2}^{(2)}\left(\mathrm{x}_{1}\right)=-\mathrm{R}_{2}^{\mathrm{f} *} \\
& \operatorname{EIv}_{3,111}^{(2)}\left(\mathrm{x}_{1}\right)+\rho \mathrm{I}\left(\Omega+\omega^{\prime}\right)^{2} \mathrm{v}_{3,1}^{(2)}\left(\mathrm{x}_{1}\right)- \\
& -\operatorname{EAv}_{1,1}^{(2)}\left(\mathrm{x}_{1}\right) \mathrm{v}_{3,1}^{(2)}\left(\mathrm{x}_{1}\right)+\mathrm{m}_{\mathrm{p}} \Omega^{2} \mathrm{v}_{3}^{(2)}\left(\mathrm{x}_{1}\right)=-\mathrm{R}_{3}^{\mathrm{f} *} \\
& \operatorname{EIv}_{3,11}^{(2)}\left(\mathrm{x}_{1}\right)+\mathrm{J}_{0}\left(\Omega+\omega^{\prime}\right)^{2} \mathrm{v}_{3,1}^{(2)}\left(\mathrm{x}_{1}\right)=-\mathrm{MR}_{2}^{\mathrm{f}^{*}} \\
& \operatorname{EIv}_{2,11}^{(2)}\left(\mathrm{x}_{1}\right)-\mathrm{J}_{0}\left(\Omega+\omega^{\prime}\right)^{2} \mathrm{v}_{2,1}^{(2)}\left(\mathrm{x}_{1}\right)=\mathrm{MR}_{3}^{\mathrm{f} *}
\end{aligned}
$$




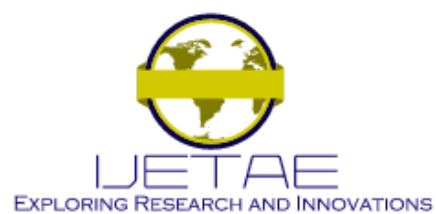

International Journal of Emerging Technology and Advanced Engineering Website: www.ijetae.com (ISSN 2250-2459, ISO 9001:2008 Certified Journal, Volume 10, Issue 07, July 2020)

\section{CONCLUSIONS}

- Insertion of external strains concerns the movement initial conditions and the geometric configuration of the main spindle.

- At balance, the initial conditions regard the axial cutting force and the bending moments determined for the main characteristic points.

- Establishing the final form of the movement equations and the balance conditions creates the premises for the stability analysis of the system composed of the main spindle and abrasive tool.

\section{REFERENCES}

[1] Bolcu, D. and Rizescu, S. 1995. La deduction du modele mathematique pour la mouvement spatial des bares composites l'aide du principe variational de Hamilton. In Annals of Craiova University, Mechanics II, 88-89.

[2] Chen, C.H., Wang, K.W. and Shin Y.C. 1994. An Integrated Approach Toward the dynamic Analysis of High-Speed Spindles System Model. J. Sound Vibration, 506-512.

[3] Chen, C.H., Wang, K.W. and Shin, Y.C. 1994. Dynamics Under Moving End Load. J. Vibrations Acoustics 116, 514-522.

[4] Moraru, V. and Ispas, C. 1982 Vibrations and Machine Tools Stability. EDP.

[5] Popescu, D. 2000. Determining The Balance Configuration, In Case Of The Oscillating Movement Of The Main Spindle At Internal Grinding. In ICMAS 2000, 113-118. 